\title{
Analysis of clinical trials and off-label drug use in hospitalized pediatric patients
}

\author{
Tamara Roldán, M.D. a, Elena Villamañán, M.D. ${ }^{a}$, Margarita Ruano, M.D. ${ }^{a}$, Yolanda Larrubia, M.D. ${ }^{a}$, \\ Pilar Gómez-Salcedo, M.D. ${ }^{a}$, and Alicia Herrero, M.D. ${ }^{a}$
}

\begin{abstract}
Introduction. The lack of pediatric clinical trials (PCTs) leads to an off-label drug use (OLDU) in children. Our objective was to analyze the number and design of PCTs and OLDU in children in the past years.

Population, material and methods. Observational and retrospective study on PCTs and OLDU in children, conducted from 2007 to 2012 in a 252-bed children's hospital. The number and design of PCTs and OLDU in children were analyzed by year and by characteristics.

Results. Eighty-seven PCTs and 449 active ingredients corresponding to 1049 drugs prescribed to hospitalized children were evaluated.Of these, $117(26 \%)$ were used off-label. The number of PCTs increased from 2008 to 2011. In 2011, 52.2\% of PCTs were non-randomized and uncontrolled studies, and only $39.1 \%$ were randomized, controlled trials. Of all studied drugs, $77 \%$ corresponded to off-label use. OLDU in children remained steady throughout the study period.

Conclusions. In our hospital, the number of pediatric research studies has increased in the past years, being non-randomized and uncontrolled studies the most frequent. OLDU in children has not changed.

Key words: research, clinical trials, off-label drug use.
\end{abstract}

http:/ / dx.doi.org/10.5546/aap.2014.eng.249

\section{INTRODUCTION}

Drugs are usually prescribed to children without having been first studied in clinical trials in this age group. ${ }^{1}$ According to several studies, up to $70 \%$ of drugs do not include enough data in their package insert (PI) regarding the pediatric age. $^{2}$

Between $36 \%$ and $100 \%$ of hospitalized children are treated with off-label drugs, ${ }^{3}$ newborn infants and infants younger than two years old account for the groups that most commonly receive drugs in this manner. ${ }^{4}$

a. Department of Pharmacy. Hospital Universitario La Paz, Madrid, Spain.

E-mail Address:

Elena Villamañán, M.D.: evillabueno@telefonica.net

Conflict of Interest: None.

Received: 12-17-2013

Accepted: 2-3-2014
In spite of this, pediatric clinical trials (PCTs) are scarce. Hurdles for their development include difficulties to conduct randomized and controlled studies, ethical issues and a lower proportion of patients. ${ }^{5}$

In addition, in many cases, the pharmaceutical industry is not interested in making investments in PCTs because of their high cost and the small proportion of the population to which they are targeted. ${ }^{6}$

In this context, and with the purpose of encouraging pediatric drug research, several health institutions have implemented new initiatives. In this sense, the World Health Organization has issued resolution WHA60.20, ${ }^{7}$ which urged its 163 Member States to improve the availability of drugs for children. The USA enacted a new act (the Pediatric Research Equity Act) in 2003, which is currently in force; ${ }^{8}$ while the European Parliament published the Paediatric Regulation in $2006 .{ }^{9}$

The objective of this study was to assess the number and design of PCTs and off-label drug use in hospitalized children over the past years.

This was an observational, retrospective study conducted between 2007 and 2012. The study was developed in a public, tertiary-care university hospital of Spain, located in Madrid, which comprises a 252-bed children's hospital with 28 subspecialties where approximately 9000 children are admitted each year.

All PCTs undertaken at the site and all drugs prescribed to hospitalized children in the study period were included. The number and design of PCTs by year and the number of noninvestigational and off-label drugs used to treat hospitalized children by year were analyzed.

In relation to PCT characteristics, the following domains were collected, as indicated in the approval document of the European Medicines Agency (EMA): age range, design characteristics (study phase, open-label or blind, randomized or not-randomized, controlled or uncontrolled), drugs involved, and pediatric specialties.

In order to establish the conditions of use of prescribed drugs, three categories were determined according to the indications provided in the PI: 
1) not assessed (effectiveness and safety in the pediatric population has not been established);

2) not authorized (contraindicated in the pediatric population);

3) not specified (the PI includes no specific information regarding the pediatric population).

The assessment also included the type of offlabel drugs indicated to children and the specialties.

Sources of data for this study included the hospital's clinical trial management software, an ad hoc application created by the site's IT Unit, which collects clinical trial characteristics according to the EMA authorization registry, and FarmaTools ${ }^{\circledR}$ (version 2.5), an electronic prescription (EP) software used to assess drug use in hospitalized children. This software records the population to which the drug is indicated, the dose used, the dosing interval or the indication, among other data.

The study was authorized by the site's Clinical Research Ethics Committee.

\section{RESULTS}

Eighty-seven PCTs and 449 active ingredients were assessed, which corresponded to 1049 marketed drugs prescribed to hospitalized children. Of these, 117 (26\%) corresponded to OLDU in children.

The number of PCTs increased from 2008 to 2011 and accounted for almost $20 \%$ of all clinical trials conducted at the site, which included both adults and children (Figure 1). In the same study period, OLDU in children remained stable and always above 20\%, as shown in Figure 2.

Analyzed PCTs were mostly phase III studies $(64.4 \%)$, followed by phase II studies (23\%). In relation to their main characteristics, they were open-label $(71 \%)$, not randomized $(58.1 \%)$ and uncontrolled (61.3\%). Up to 2008, randomized, blinded and controlled studies were more prevalent than open-label, non-randomized and uncontrolled trials, reaching $57.1 \%$ of all PCTs.

Between 2008 and 2009, the trend shifted, and open-label, non-randomized and uncontrolled studies increased, although the advancement of both design types remained globally stable. Towards the end of the study period, the number of both types of PCTs was fairly similar, accounting for $33.3 \%$ and $26.7 \%$ of all PCTs, respectively (Figure 3 ).

In relation to drugs studied in PCTs, $77 \%$ were used off-label and $23 \%$ corresponded to nonmarketed investigational drugs. Most study drugs were antiinfective agents (34.5\%), antineoplastic agents and immunomodulators (20.7\%), and cardiovascular drugs (11.5\%). The evolution of PCTs in these therapeutic groups over the study period is shown in Figure 4.

The two pediatric specialties that conducted most PCTs were hematooncology (19.5\%) and neonatology $(11.5 \%)$. All PCTs included in the study were multicenter and sponsored by the pharmaceutical industry.

FIGURE 1.Ratio between pediatric clinical trials and total clinical trials undertaken at the Pharmacy Service

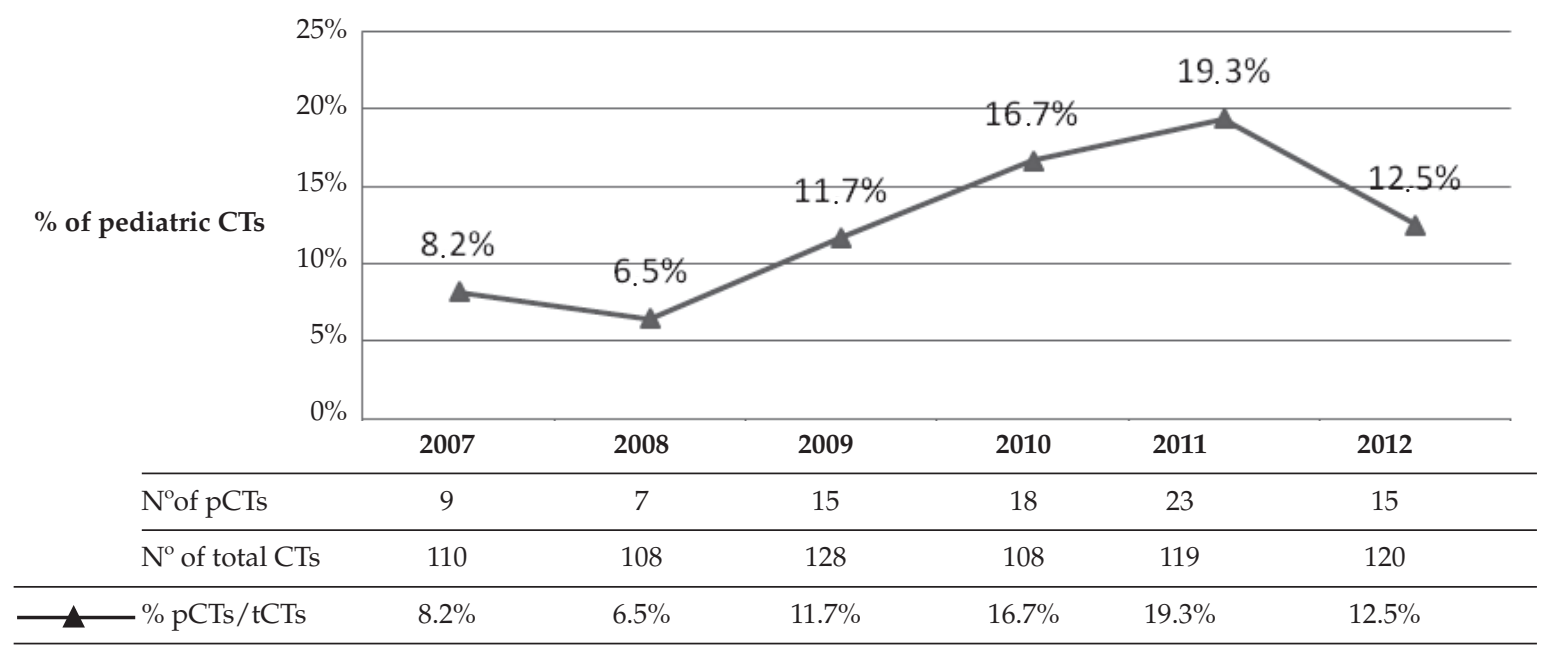

CTs: clinical trials; pCTs: pediatric CTs; tCTs: total clinical trials. 
When analyzing the conditions of pediatric drug use, we observed that $80.8 \%$ of drugs corresponded to category $a$ (not assessed/not recommended; effectiveness and safety in the pediatric population has not been established), $15.4 \%$ corresponded to category $b$ (not authorized; contraindicated in the pediatric population), and $3.8 \%$ corresponded to category $c$ (not specified in the PI; there is no specific information regarding the pediatric population).

Most drugs routinely used in pediatrics corresponded to the treatment of digestive tract conditions $(25.6 \%)$, infections $(21.8 \%)$ and nervous system disorders (16.5\%). However, the therapeutic groups most commonly used off-label were hypnotics and sedatives (lormetazepam and clomethiazole; $5.73 \%$ ), antithrombotic agents (enoxaparin, dipyridamole and bemiparin; 2.95\%) and antibacterial drugs (daptomycin; 1.27\%).

In terms of pediatric specialties, the ones that issued most off-label prescriptions were general pediatrics $(28.8 \%)$, pediatric surgery $(19.5 \%)$, liver and kidney transplant (15.5\%), hematooncology $(11.0 \%)$, and neonatology $(4.7 \%)$.

FIGURE 2. Evolution of drug use by conditions of use

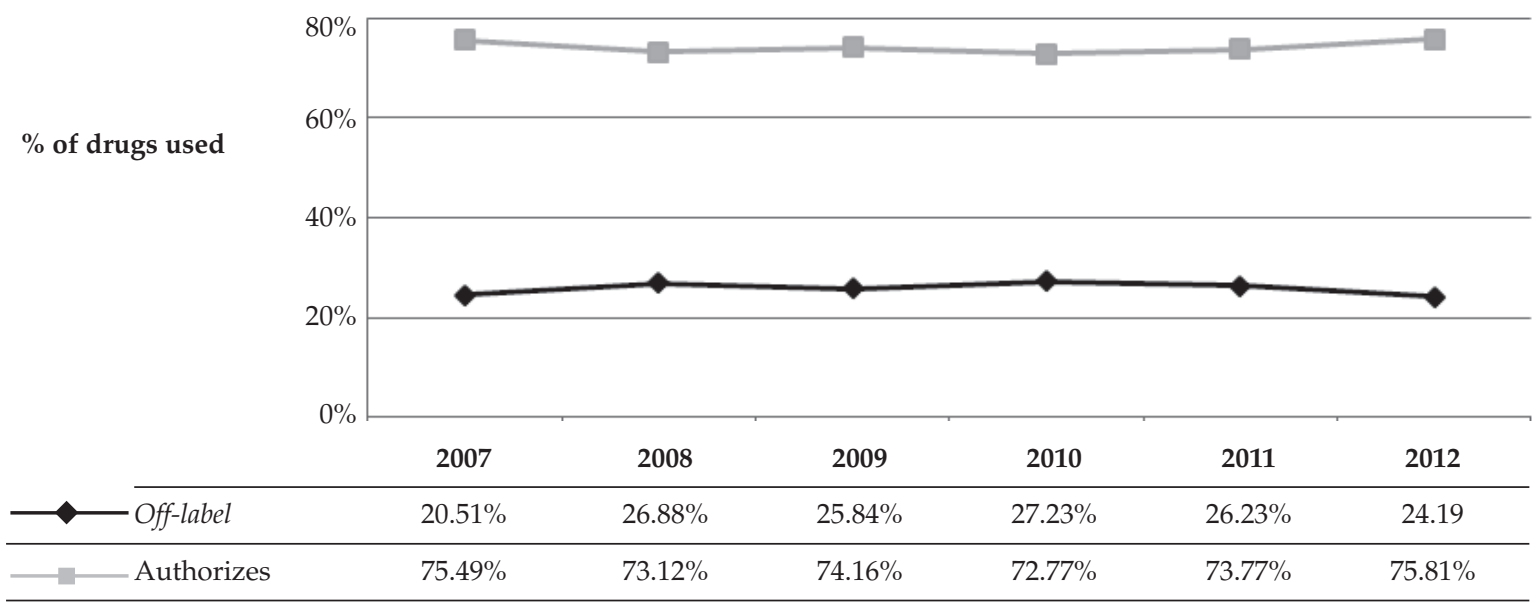

Figure 3. Evolution of clinical trials in terms of design

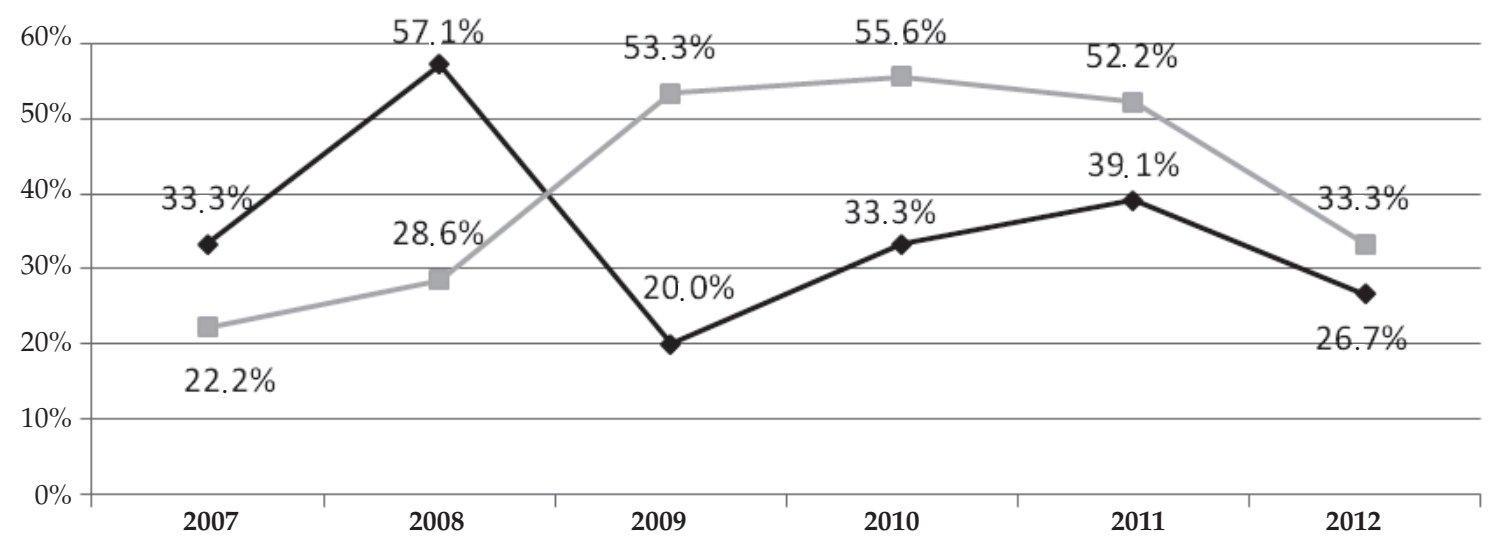

$\%$ of randomized, blind, controlled studies.

\% of open-label, non-randomized, uncontrolled studies. 
FIGURE 4. Evolution of pediatric clinical trials in terms of therapeutic group

10

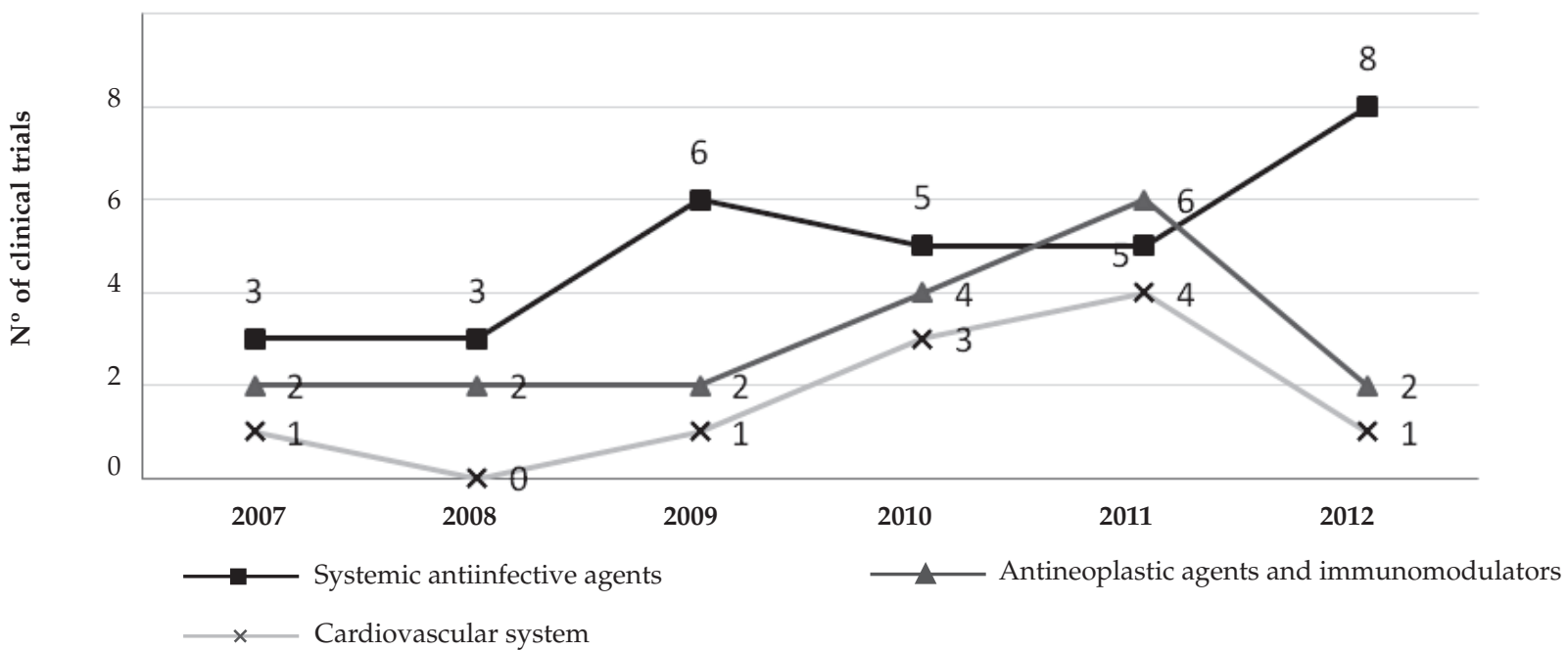

\section{DISCUSSION}

Based on our results, and consistently with otherstudies, ${ }^{10}$ the number of PCTs increased from 2007 to 2011, which may be related to the 2006 European publication of new initiatives to encourage pediatric drug research. ${ }^{9}$

However, off-label drug use in pediatrics remained sort of stable throughout the study. Our results indicate that more than $25 \%$ of drugs were used this way, basically due to the lack of pediatric studies, as specified in their PIs. Other authors have obtained consistent results and have detected similar, and even higher rates. ${ }^{11,12}$

In terms of design, it is worth noting that PCTs have remarkably changed over the past years at our site: they went from being mostly randomized, controlled studies to almost the same number as open-label, non-randomized and uncontrolled studies. It is known that randomized and controlled clinical trials are the best tools to assess the effectiveness and safety of a treatment, but open-label uncontrolled studies are less complex and require a smaller financial investment. In this sense, research incentives could have had a positive effect in quantitative terms, but their impact was negative in terms of quality.

In line with other studies, ${ }^{13}$ most drugs used in PCTs were antiinfective agents, which in addition are considered a pediatric research drug priority by the EMA. ${ }^{14}$

Hematooncology and neonatology were the two specialties that conducted most PCTs, probably due to the severity of conditions and the lack of therapeutic alternatives available for these patients.

Most commonly prescribed drugs were for the treatment of digestive tract conditions and infections, mainly beta-lactam antibiotics (penicillins and cephalosporins). These data are consistent with other previously published. ${ }^{15}$

Conversely, the most commonly drugs used offlabel were, firstly, hypnotics and sedatives, secondly, antithrombotic agents, and thirdly, antibiotics.

\section{Study limitations}

First of all, there are limitations resulting from the study design because this is a retrospective and observational study.Secondly, we only analyzed clinical trials exclusively conducted on children, leaving out those that included both adults and children. Lastly, manual prescriptions using nurse sheets were not analyzed due to lack of registry in the EP software.

\section{CONCLUSION}

In our site, the number of PCTs has increased since 2007; most of them looked into drugs that are already available and that have previously been used off-label. Non-randomized and uncontrolled studies were the most common designs.In addition, OLDU in children remained stable.

\section{REFERENCES}

1. Shah SS, Hall M, Goodman DM, Feuer P, et al. Off label drug use in hospitalized children. Arch PediatrAdolesc Med2007;161(3):282-90.

2. Shah S, Whittle A, Wildford B, Gensler G, Wenler D. 
How do institutional review boards apply the federal risk and benefitstandards for pediatric research? JAMA 2004;291(4):476-82.

3. NeubertA, Wong IC, Bonifazi A, Catapano M, etal. Defining off-label and unlicensed use of medicines for children: Results of a delphi survey. Pharmacol Res 2008;58(5-6):31622.

4. Giglio ND, Malozowski S. Prescripciones fuera de prospecto. Arch Argent Pediatr 2004;102(2):121-4.

5. Li JS, Cohen-Wolkowiez M, Pasquali S. Pediatric cardiovascular drug trials, lessons learned.JCardiovascPharmacol 2011;58(1):4-8.

6. Fernández-Llamazares CM, Manrique-Rodríguez S, Sanjurjo-Sáez M. Seguridad en el uso de medicamentos en pediatría. Arch Argent Pediatr 2011;109(6):510-8.

7. World Health Assembly (ed.). Resolución WHA60.20: Better medicines for children. Ginebra, Suiza: World Health Organization;2007. [Accessed on:February4,2014]. [Available at: http:/ / www.who.int/childmedicines/publications / WHA6020.pdf].

8. The Pediatric Research Equity Act of 2013, S.650. 108th Congress of the United States of America, first session, Washington, January 2003.

9. Reglamento del Parlamento Europeo y del Consejo sobre Medicamentos para Uso Pediátrico, 1901/2006(12 de diciembre, 2006). [Accessed on: February 4, 2014].[Available athttp://www.ub.edu/legmh/disposici/reg1901.htm].

10. Sampson MR, Benjamin DK, Cohen-Wolkowiez M. Evidence based guidelines for pediatric clinical trials: focus on StaR Child Health. Expert Rev Clin Pharmacol 2012;5(5):525-31.

11. Hsein L, Breddemann A, Frobel AK, Heusch A, et al. Offlabel drug use among hospitalized children: identifying areas with the highest need for research. Pharm World Sci2008;30(5):497-502.

12. Knopf H, Wolf IK, Sarganas G, Zhuang W, et al. Offlabel medicine use in children and adolescents: results of population-based study in Germany. BMC Public Health 2013;13(1):631.

13. Pandolfini C, Bonati M. European paediatric research and children's therapeutic needs. A trial review. ActaPaediatr2008;97(9):1232-7.

14. Revised provisional priority list for studies into off-patent paediatricmedicinalproducts. [Accessed on: August 8, 2013]. [Available at: http://www.ema.europa.eu/ docs/en_GB/document_library/Other/2013/06/ WC500143970.pdf].

15. Santos DB, Clavenna A, Bonati M, Coelho HL. Off-label and unlicensed drug utilization in hospitalized children in Fortaleza, Brazil. Eur J ClinPharmacol2008;64(11):1111-8. 